\title{
Diabetic retinopathy through retinal image analysis: A review
}

\author{
Y.Madhu Sudhana Reddy ${ }^{1 *}$, R. S. Ernest Ravindran ${ }^{2}$, K Hari Kishore ${ }^{3}$ \\ ${ }^{1}$ Research Scholar, Department of ECE, Koneru Lakshmaiah Education Foundation, \\ Vaddeswaram, Guntur, Andhra Pradesh, India-522502 \\ ${ }^{2}$ Department of ECE, Koneru Lakshmaiah Education Foundation, Vaddeswaram, Guntur, Andhra Pradesh, India-522502 \\ ${ }^{3}$ Department of ECE, Koneru Lakshmaiah Education Foundation, Vaddeswaram, Guntur, Andhra Pradesh, India-522502 \\ *Corresponding author E-mail: ymsr1016@gmail.com
}

\begin{abstract}
In this paper, the recent advancement in the Digital Image Processing Aspects in the Diabetic Retinopathy (DR) were been discussed. The major approaches in DR are categorized into four classes namely Preprocessing, Optic Disk Detection, Blood Vessel Extraction and supervised classification. The optic disk, blood vessels and exudates gives more analytical details about the retinal image, segmentation of those features are very important. Further these approaches are classified into finer classes based on the methodologies accomplished in the respective schemes. The details of the database those used for testing the proposed algorithms is also illustrated in this paper. The details of performance metrics such as accuracy, sensitivity, specificity, precision, recall and F-measure are also discussed through their mathematical expressions.
\end{abstract}

Keywords: Diabetic Retinopathy (DR), Optic Disk, Blood Vessel Segmentation, Exudates, datasets.

\section{Introduction}

Automation in medical diagnosis is been in investigation for a longer time. Researchers have focused on various aspects of medical data to process, towards automation of medical diagnosis, resulting in faster, economical and accurate diagnosis. The primal objective of the system is to overcome the dependency of the sample analysis for the captured medical data and process in a supervised manner to extract and detect the effects observed in the given sample. Towards automation, various medical samples, such as MRI, mammogram, X-rays, ECG, EEG etc., were analyzed in either online or offline mode. Towards developing automation approach for the detection of Diabetic Retinopathy "(DR) through retinal image analysis a similar objective of retinal image diagnosis was also developed [1,2].

Generally the segmentation of blood vessel in retinal image considers the features of blood vessel such as Length of blood vessel, width of blood vessel, vessel tortuosity and branching pattern of vessel. The automatic detection of retinal vasculature can help in Evaluation of retinopathy of prematurity [3], fovea vascular region detection [4], arteriolar narrowing [5], relationship between vessel tortuosity and hypertensive retinopathy [6], vessel diameter measurement in relation with diagnosis of hypertension [7], and computer assisted laser surgery. Further the automatic extraction of branch points of vessels and maps of vessels can be used for multimodal or temporal image registration [8], optic disk identification [10] and location of fovea. Finally, the retinal vascular structure of every individual is unique which can be used for biometric identification $[11,12]$.

Manual segmentation of retinal blood vessels is a deadly task which requires skill and training. Hence there is a necessity of automatic blood vessel segmentation in retinal images to make the system automatic, which is the main aspect accepted by medical council in the development of computer assisted diagnostic for ophthalmic diseases. In earlier stages, many approaches were proposed to perform blood vessel segmentation in retinal images.

\section{A. Image oriented retina analysis}

The retinal image is captured through a specific camera called fund us camera. The fund us camera captures the inner surface of the eye which includes the retina, optic disc, macula, and posterior pole [13]. The eye's fund us is the only part of the human body where the microcirculation can be observed directly. The diameter of the blood vessels around the optic disc is about $150 \mu \mathrm{m}$, and an ophthalmoscope allows the observation of blood vessels with diameters of $10 \mu \mathrm{m}$. Medical signs that can be detected from observation of fund us eye image include hemorrhages, exudates, blood vessels, optic disk and cotton wool spots. Figure 1 shows a typical retinal image labeled with various components of DR. Micro aneurysms appear as small red dots that may lead to hemorrhage(s), while the hard exudates appear as bright yellow lesions. Generally the severity of DR is measured through the spatial distribution of exudates, and Micro aneurysms and Hemorrhages (MAHM). Hence the DR detection methodology involves the following phases; Preprocessing: Preprocessing phase involves the normalization image contrast and luminance etc. Optical Disk Segmentation: Optical disk detection algorithm uses the property of fund us image that the optical disk region is the brightest region and therefore the intensity value is the criterion used to detect optical disk. Blood Vessel Extraction: Blood vessel extractions done using morphological operations on the green channel of fund us image. Fovea Detection: Fovea Detection is detected using the location of the optic disk and curvature of the main blood vessel. Micro Aneurysms and Hemorrhages (MAHM): Hemorrhages and micro aneurysms are treated as holes and morphological filling is performed on the green channel to identify them. 


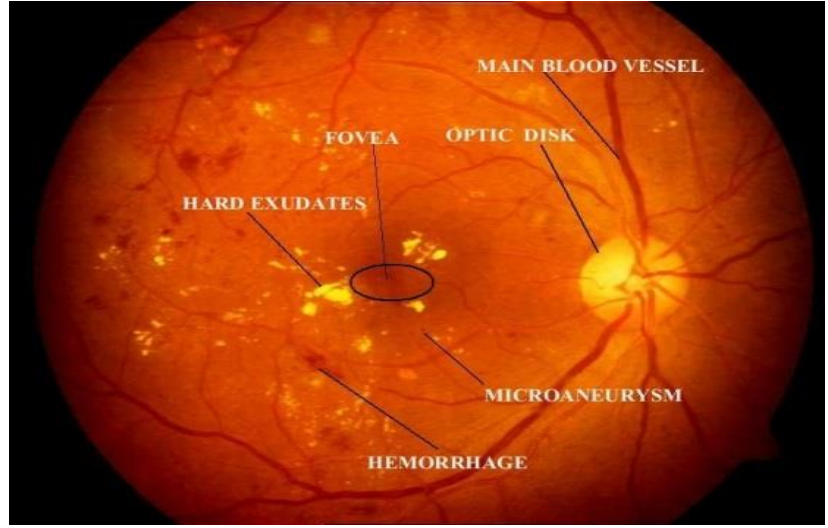

Fig.1: Morphological attributes of retinal images

The available diagnosis for Diabetic Retinopathy are: Digital Retinal Images for Vessel Extraction (DRIVE), Structured Analysis of the Retina (STARE), Automatic Retinal Image Analysis (ARIA), Image Retinopathy (IMAGERET), Methods to Evaluate Segmentation and Indexing Techniques in the Field of Retinal ophthalmology (MESSIDOR), Retinal Vessel Image set for Estimation of Widths (REVIEW) and vessel registration for a reliable computation of arteriovenous ratio (VICAVR).

The general performance metrics which are used for the performance evaluation, i.e., to measure how exactly the status of DR is identified, are as follows;

$$
\text { Accuracy }=\frac{T P+T N}{T P+T N+F P+F N}
$$

Whereas,

True Positive $(\mathrm{TP})=$ correctly identified,

False Positive (FP) = incorrectly identified,

True Negative $(\mathrm{TN})=$ correctly rejected,

False Negative $(\mathrm{FN})=$ incorrectly rejected.

Along with accuracy, to show the enhancement of propose approach and to compare the proposed approach with earlier approaches few more metrics such as sensitivity, specificity, Recall, precision and F-measure was evaluated with following mathematic expressions.

Sensitivity measures the proportion of positives that are correctly identified as,

$$
\text { Sensitivity }=\frac{T P}{T P+F N}
$$

Specificity measures the proportion of negatives that are correctly identified as,

$$
\text { Specificity }=\frac{T N}{T N+F P}
$$

Precision is the fraction of identified instances that are correct, while recall is the fraction of correct instances that are identified.

$$
\begin{aligned}
& \text { Recall }=\frac{T P}{T P+F N} \\
& \text { Precision }=\frac{T P}{T P+F P}
\end{aligned}
$$

F-measure or balanced F-score is a measure that combines precision and recall is the harmonic mean of precision and recall.

$F-$ measure $=\frac{\text { 2.Recall } . \text { Precision }}{\text { Recall }+ \text { Precision }}$

Based on the above metrics, a performance plot is drawn between sensitivity and specificity and called as Receiver Operating Characteristics (ROC) curve. A sample ROC plot is shown in figure.2.

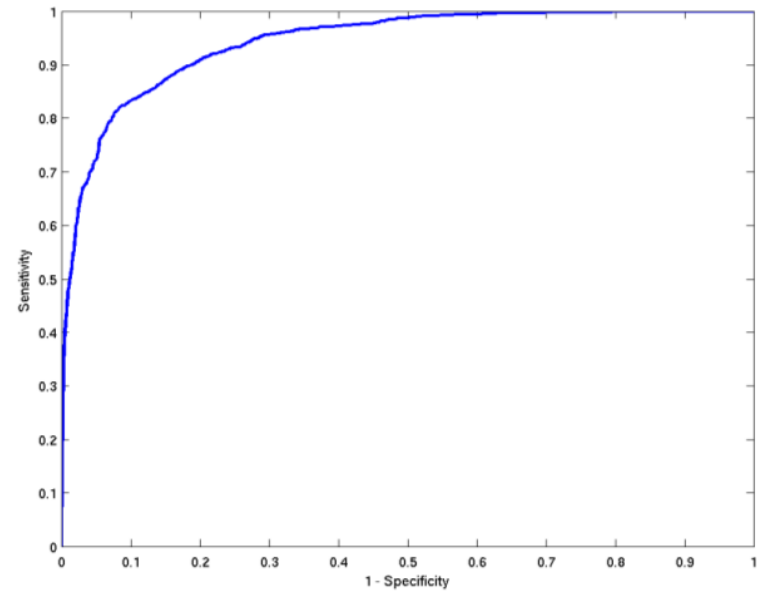

Fig.2: An example ROC curve.

\section{Approaches}

\section{A. Preprocessing}

Preprocessing technique is to attenuate image variation by normalizing the original retinal image against a reference model or data set for subsequent viewingand processing or analysis. Because of the acquisition process, very often retinal images are non-uniformly illuminated and exhibit local luminosity and contrast variability. This problem may seriously affect the diagnostic process and its outcome, especially if an automatic computer-based procedure is used to derive diagnostic parameters. A new method is proposed by For acchia $M$ et.al, and Ehsan $S$ [14,15] to normalize luminosity and contrast in retinal images, both intra and inter image. The method is based on the estimation of the luminosity and contrast variability in the background part of the image and the subsequent compensation of this variability in the whole image. Due to the complex imaging setup, there is a large luminosity and contrast variability within and across images. Here, Gopal Dutt Joshi [16] uses the knowledge of the imaging geometry and proposed an enhancement method for color retinal images, with a focus on contrast improvement with no introduction of artifacts. The method uses non-uniform sampling to estimate the degradation and derive a correction factor from a single plane. Gopal Dutt Joshi [16] also proposed a scheme for applying the derived correction factor to enhance all the color planes of a given image.Furthermore, the empirical observations were carried out by Shin D.S et.al.,[17] and Leandro J.J.G et.al., [18] to identify the green channel of RGB images as containing the maximum contrast. Rapantzikos K et.al., [19] also note that the green channel appears to provide more information and is less subject to non-uniform illumination and blue channel contains little information for the detection of retinal features.

Histogram equalization redistributes the histogram of each color channel in the input image such that the output image contains a uniform pixel value distribution. Hani [20] proposed an improved nonlinear hue-saturation-intensity color model (INHSI) to preserve color information of the retinal images. The intensity component is enhanced by Rayleigh transformation in contrast-limited adaptive histogram equalization (Rayleigh CLAHE) [21,23] algorithm. Redistributing the histogram to match that of a reference image, which does not necessarily contain the lesion, may obscure evidence of the pathology.

Contrast enhancement technique has an important role in the field of retinal image enhancement as it improves the contrast of an image. The contrast is an important factor to differentiate a good quality image from a low-quality image. A new retinal image contrast enhancement method for Diabetic Screening System is presented by N.S.Datta [22]. This method is compared with different contrast enhancement approaches [24, 25] and found that it produces better image quality and preserves the mean brightness of the input images which is very important for medical image analysis.Rapantzikos K et.al.,[19] Proposed a multilevel histogram 
equalization (MLE) method as a preprocessing step in the detection of drusen. The approach is based on the sequential application of histogram equalization to progressively smaller nonoverlapping neighborhoods. However, the detection of multiple types of anatomy and pathology with different physical dimensions is also problematic when relying upon a hierarchal neighborhood method.

\section{B. Optic Disk (OD) segmentation}

Segmentation of the optic disk usually refers to the subsequent task of determining the contour of the disk. Localization and segmentation of the optic disk are important tasks in retinal image analysis. The disk center and contour are often prerequisite landmarks in establishing a frame of reference for identifying Diabetic Retinopathy. Related work regarding the OD detection using fund us images can be broadly categorized into two groups based on shape, color and structure of the OD and the properties of the retinal vasculature. The automated algorithms belonging to both the categories are discussed as follows.

\subsubsection{Structural features based OD detection}

This category includes the well-known methods that rely on the appearance and size of the OD [26], such as the method in [27] that detects the bright regions by mathematical morphology and detects the OD as the largest high contrast average circular-shape area. The method in [28] uses morphology followed by watershed transform for the model-based OD detection. The method in [42] first locates a pixel belonging to the OD region, and then applies boundary segmentation by morphology, edge detection, and circular Hough transforms. Other well-known methods in [29] and [30] use a line operator to capture the circular brightness structure of the OD since the maximum and minimum variation along the linear operator has a specific pattern to locate the OD.

\subsubsection{Retinal Vasculature based OD detection}

This category of methods examines the retinal vasculature for locating the OD, since the OD is the point of entrance of the optic nerve and blood vessels which branch out into finer vessels through the retina. The method in [26] applies a search on the branching network patterns formed by the blood vessels to converge to the region where most paths ended, followed by the application of Hough transform on all such regions to finally locate the OD. Another method in [31] matches directional patterns of the retinal blood vessels to match the direction of OD vicinity. The method described by Rangayyan $R$ et.al., [32] localizes the OD by tracking the blood vessels using Gabor filters to detect the peaks in nodes via phase portrait analysis and locates the OD at the focal point of the vessels using Hough transform. Welfer D et.al., [33] applied watershed transform for detecting the OD location and disc boundary using the information regarding the major vessel arcade. Besides, maximization of vessel patternbased entropyis used to detect the location of the OD in [34].

\subsubsection{Combined strategies}

Some algorithms, however, have combined the two categories, such as the method in [35] that locates the OD based on the structure of the OD, the convergence of blood vessels at the OD, and the variation in the size of the blood vessels entering the OD. Another method in [36] uses the feature-based k-nearest neighbor classifier for training and extracting the OD. The method in [43] uses an ensemble of pyramidal decomposition, edge detection, entropy-filter-based detection, Hough transform, and featurevector-based algorithms for detecting the OD. Another method in [37] uses the principal component image plane followed by the removal of vessel regions, image in painting, stochastic watershed transform, and regional discrimination for the OD boundary detection. Also, the method in [44] applies super-pixel classification to separate pixels of the disc from non-discs using histogram and center surround statistics.

\subsubsection{Other Methods}

The recent works in [38] and [39] separate the vascular information from the in painted vessel removed images and use the radial-symmetry-based OD location strategy. In [38], the OD region is selected as the one having high vesselness in its vicinity, while the method in [39] uses complex contour fitting at increased resolution using in painted images and vessel masks. Another recent work in [40] uses three vessel distribution features: Local vessel density, compactness, and uniformity to find horizontal OD coordinate, followed by Hough Transform and parabola fitting to find the vertical OD coordinate. A set of morphological opening and closing operations are carried out in the method proposed by Marin D et.al., []41 to produce an image with bright regions followed by blood vessel confluence detection at the OD and circular Hough transform.

\section{Retinal blood vessel extraction}

The segmentation and measurement of the retinal vessels is of primary interest in the diagnosis and treatment of a Diabetic Retinopathy. As previously discussed, the accurate segmentation of the retinal blood vessels is often an essential prerequisite step in the identification of retinal disorders and abnormalities. The vessel cross-sectional intensity profiles approximate a Gaussian shape or a mixture of Gaussians in the case where a central vessel reflex is present. The orientation and grey level of a vessel does not change abruptly; they are locally linear and gradually change in intensity along their lengths. The vessels can be expected to be connected and, in the retina, form a binary treelike structure. However, the shape, size and local grey level of blood vessels can vary hugely and some background features may have similar attributes to vessels. Vessel crossing and branching can further complicate the profile model. As with the processing of most medical images, signal noise, drift in image intensity and lack of image contrast pose significant challenges to the extraction of blood vessels. In earlier so many approaches are proposed to perform retinal vasculature segmentation based on various aspects like curvature of vessel, morphological operations, matched filtering etc. This section gives a complete knowledge about the earlier proposed retinal vascular segmentation approaches.

\subsubsection{Curvature based methods}

In this case, the blood vessel can be modeled as a curve for smoother extraction. Along with this, the abnormalities in the blood vessels can be determined through the tortuosity of the vessels. Tortuosity is an indication of how winding a blood vessel is [45]. If there is more tortuosity in the blood vessel there may be chance of DR. Tortuosity can be happen in small portions of blood vessel or throughout the blood vessel network.

\subsubsection{Arc length over chord length ratio methods}

Methods of this group have simple mathematical expressions. The first methods of this category were introduced in [46] and were widely utilized thereafter [47-50]. However, it is apparent that the arc over chord length ratio, on its own, is insufficient for determination of vessels with smooth curvature and vessels with variation in curvature direction. For compensation, [51] and [52] proposed modifications on the approach. In [52], vessels are partitioned into segments with the same convexity and a weighted sum of arc over chord length of all segments is proposed as a tortuosity measure.

\subsubsection{Methods based on curvature}

Curvature is a mathematical measure for how inflected a curve is at a certain coordinate. Hart W.E et.al.,[48] used curvature method 
to develop two tortuosity measures which are the integral of curvature and the integral of curvature squared. Moreover, the ratio of these integrals over arch or chord length has also been proposed as tortuosity measures in [48]. In [53], the integral of squared curvature derivative is suggested as a measure of tortuosity. These or other curvature-based algorithms have been used in most of the recent works including [54], [55] as well. Curvature based tortuosity measures are more reliable, but they impose a heavy computational burden compared to the methods of the first group.

\subsubsection{Methods based on angle variation}

These methods compute the direction variations of the vessel to measure tortuosity. In [56], the average of the angles between sample center points that describe the vessel (called local direction variation) was used to measure tortuosity. In [57], the same method is used to measure local angles and the number of times a local angle surpasses $30^{\circ}$ is considered as tortuosity index.

\subsubsection{Methods based on another domain}

Instead of extracting the curvature of blood vessel in spatial domain, the curvature of blood vessel is calculated in the transform domain. Kaupp A et.al.,[58] Used Fourier analysis and [59] used circular Hough transform to calculate curvature. Moreover, in [60], Non-Subsample Contour let Transform is used for curvature calculation. The key feature of these methods is evaluation of tortuosity without vessel extraction. However, they suffer from heavy computational burden as well. There have also been some special cases of tortuosity measurement algorithms. For example, [61] used cubic-spline interpolation for measuring tortuosity.

\subsubsection{Morphological based methods}

Morphological processing which consist of techniques dealing with digital image processing using mathematical morphology by applying some structure element (SE) to binary images and sometimes to gray-level images.

A novel three-stage blood vessel segmentation algorithm was developed in [62]. The first stage is pre-processing by high-pass filtering then extracting a binary image and another binary image is reconstructed from morphologically enhanced image for the vessel regions. Next the major vessels are extracted which is common regions from these two images. Then the second stage, Gaussian Mixture Model (GMM) classifier is used to classify all pixels in the two binary images which are remained from previous stage. Morphological multi-scale enhancement method is also presented in [64]. For the extraction of the blood vessels in the angiogram; fuzzy filter and watershed transformation are used. In the method [65] an approach is presented which is combined unique vessel centerlines detection with morphological bit plane slicing. The first order derivative of a Gaussian filter is used in four directions to extract the centerlines, and then performing an average derivative and derivative signs with the extracted centerlines. Morphological multidirectional top-hat operation is applied on blood vessels gray-scale image with linear structure element to obtain the orientation map and shape. In [66] fast discrete curve let transform with multi-structure mathematical morphology is proposed. For detecting the blood vessels edges, multi-structure morphological transformation is applied. Then morphological opening is applied on the result image to remove the false edges. An automated enhancement and segmentation method for blood vessels is presented in [67]. This method decreases the optic disc influence and emphasizes the vessels by applying a morphological multidirectional top-hat transform with rotating structuring elements to the background of the retinal image.

\subsubsection{Matched Filtering Methods}

Matched filtering for the detection of the vasculature convolves a 2D kernel with the retinal image. The kernel is designed to model some feature in the image at some unknown position and orientation, and the matched filter response (MFR) indicates the presence of the feature. Various approaches [71-73] are proposed in earlier based on matched filtering.

Al-Rawiet.al., [72] improved Chaudhuriet.al.,'s [71] matched filter (MF) by using an exhaustive search optimization procedure to find the best parameters for matched filter size, the standard deviation and threshold value. The improved MF outperforms Chaudhuri's classical parameter matched filter. Yao and Chen [73] uses a 2-D Gaussian matched filter for retinal vessel enhancement and then a simplified pulse coupled neural network is employed to segment the blood vessels by firing neighborhood neurons. Next, a fast 2D-Otsu algorithm is used to search the best segmentation results. Due to the Gaussian nature of blood vessel profile, the MF with Gaussian kernel often misclassifies non-vascular structures as vessels. To avoid such false detection, [68] introduces Laplacian of Gaussian (LoG) filters in the vessel segmentation process. In [69], a novel matched filter based on a new kernel function with Cauchy distribution is introduced to improve the accuracy of the automatic retinal vessel detection compared with other available matched filter-based methods, most notably, the methods built on Gaussian distribution function. [70] Represents a new matched filter-based vasculature segmentation method for 2-D retinal images. First, a raw segmentation is acquired by thresholding the images preprocessed using weighted improved circular Gabor filter and multi-directional multi-scale second derivation of Gaussian. After that, the raw segmented image is fine-tuned by a set of novel elongating filters.

\section{Supervised classification}

In supervised method, the rule for vessel extraction is learned by the algorithm based on a training set of manually processed and segmented reference images often termed as the gold standard. This vascular structure in these ground truth or gold standard images is precisely marked by an ophthalmologist.

Artificial Neural Network is one of the most prominent techniques for supervised classification and most of the applications accomplished it. An ANN based vessel segmentation approach is developed in [74] based Self-Organizing Map (SOM). Firstly, a multidimensional feature vector is constructed with the green channel intensity and the vessel enhanced intensity feature by the morphological operation. Secondly, self-organizing map (SOM) is exploited for pixel clustering, which is an unsupervised neural network. Finally, we classify each neuron in the output layer of SOM as retinal neuron or non-vessel neuron with Otsu's method, and get the final segmentation result. In [75] a supervised classification technique was proposed based on the Neural Networks and grey-level co-occurrence matrix. The required specific features are extracted through the grey-level cooccurrence matrix and they are trained through ANN. [76] proposed a supervised segmentation technique that uses a deep neural network trained on a large sample of examples preprocessed with global contrast normalization, zero-phase whitening, and augmented using geometric transformations and gamma corrections. Several variants of the method are considered, including structured prediction, where a network [77] classifies multiple pixels simultaneously.

In [78], we present a novel method to segment retinal blood vessels to overcome the variations in contrast of large and thin vessels. This method uses adaptive local thresholding to produce a binary image then extract large connected components as large vessels. The residual fragments in the binary image including some thin vessel segments (or pixels), are classified by Support Vector Machine (SVM).[79]Presents a new supervised method for blood vessel detection in digital retinal images. This method uses a Fuzzy logic based Support Vector Machine scheme for pixel organization and computes a 5-D vector composed of gray-level 
and intensity histogram-based features for pixel representation. The combination of several image processing techniques with SVM classification for vessel segmentation is proposed in [80]. The combination of the radial projection and the semi-supervised self-training method using SVM is employed by [81] for vessel segmentation. [82]Present an automated segmentation method for blood vessels in images of the ocular funds. The method uses a supervised classification of vessels at each pixel based on its feature vectors. The feature vectors include the responses of the pixel to the multi-scale vessel enhancement filtering and Gabor filtering at multiple scales and multiple orientations. We use a support vector machine to extract the vessels. [83] Proposed the radial projection method to locate the vessel centerlines. Then the supervised classification is used for extracting the major structures of vessels. The final segmentation is obtained by the union of the two types of vessels after removal schemes.

As per the above discussions the al earlier approaches are categorized into preprocessing base, OD segmentation based, Blood vessel extraction based and supervised classification based approaches. They can be further classified into various categories based on the detection of inner details such as detection of exudates, fovea and macula localization, Detection of micro aneurysms or hemorrhages, Detection of retinal exudates and cotton wool spots etc. [89-94] and classified as vessel profile model based approaches [98-100], vessel tracking [95-97] based approaches, pixel based classification approaches. Further a fine classification can also be carried out by observing the visual characteristics of optic disk, localization of OD and segmentation of OD. Opposite to the supervised classification, i.e., unsupervised classification based approaches like Fuzzy c-means clustering $[84,87]$ based vessel segmentation approaches, Bayesian image analysis [85], Radius based Clustering Algorithm (RACAL) [88] which uses a distance based principle to map the distributions of the image pixels.

Among the above discussed approaches, the performance of algorithms based on supervised classification is better in general than their counterparts. The performance of almost all the approaches is measured through Received Operating Characteristics (ROC) and it is found to be approximately 0.95 . However, the supervised classification based algorithms do not work well under the image with non-uniform illumination variations; they produce false detection in some images on the border of optic disk. For the blood vessel segmentation matched filter base approaches are observed to be more efficient and achieved an optimal accuracy. The matched filtering alone cannot handle vessel segmentation in pathological retinal images; therefore, it is often employed in combination with other image processing techniques.

\section{Conclusions}

A structured survey of algorithms for the automatic detection of Diabetic Retinopathy in digital retinal images has been presented. The development of an effective tool for incorporation into diabetic retinopathy screening programmers is a highly desirable goal. An efficient system that can routinely analyze digital retinal images and eliminate those where no new or increased pathology is present, would significantly reduce the workload for ophthalmologists and graders in diabetic retinopathy screening centers. This review has focused completely on the analysis of digital images of the retina in the field of diabetic retinopathy. The process of analyzing the digital image of retina is may be viewed as a series of steps, for each of which, a choice of technologies or algorithms is available. In this survey, the diversity of the identified studies made it impossible to conduct a full quantitative statistical analysis of the performance of each processing step within the various approaches.

The qualitative analysis allowed articles to be grouped according to the methodological approaches used for each step in the process, and trends within the literature to be identified. It is observed that in most cases, the existing methodologies have some shortcomings" and many of the articles set out to develop improved methodologies derived from an existing technique.

\section{References}

[1] SangmeshBiradar, A.S.Jadhav, "A Survey on Blood Vessel Segmentation and Optic Disc Segmentation of Retinal Images", International Journal of Advanced Research in Computer and Communication Engineering Vol. 4(5), 2015.

[2] T. Teng, M. Lefley, D. Claremont, "Progress towards automated diabetic ocular screening: a review of image analysis and intelligent systems for diabetic retinopathy", Medical and Biological Engineering and Computing Vol.40, pp.2-13, 2002.

[3] C. Heneghan, J. Flynn, M. O'Keefe, M. Cahill, "Characterization of changes in blood vessel width and tortuosity in retinopathy of prematurity using image analysis", Medical Image Analysis, Vol.6, pp.407-429, 2002.

[4] Haddouche, M. Adel, M. Rasigni, J. Conrath, S. Bourennane, "Detection of the foveal avascular zone on retinal angiograms using Markov random fields", Digital Signal Processing,Vol.20, pp.149-154, 2010.

[5] "E. Grisan, A. Ruggeri", "A divide et impera strategy for automatic classification of retinal vessels into arteries and veins", Proceedings of the 25th IEEE Annual International Conference in Engineering in Medicine and Biology Society, vol. 891, pp. 890 893, 2003"'.

[6] M. Foracchia, "Extraction and quantitative description of vessel features in hypertensive retinopathy fundus images", 2nd International Workshop on Computer Assisted Fundus Image Analysis, 2001

[7] "J. Lowell, A. Hunter, D. Steel, A. Basu, R. Ryder, R.L. Kennedy", "Measurement of retinal vessel widths from fundus images based on 2-D modeling", IEEE Transactions on Medical Imaging, Vol.23, pp.1196-1204, 201.

[8] F. Zana, J.C. Klein, "A multimodal registration algorithm of eye fundus images using vessels detection and Hough transform", IEEE Transactions on Medical Imaging,Vol.18,pp.419-428, 1999.

[9] "K. Fritzsche, A, Can, H. Shen, C. Tsai, J. Turner, H.L. Tanenbuam, C.V. Stewart, B. Roysam, J.S. Suri, S. Laxminarayan, "Automated model based segmentation, tracing and analysis of retinal vasculature from digital fundus images", in: State-of-TheArt Angiography, Applications and Plaque Imaging Using MR CT Ultrasound and X-rays, Academic Press, pp. 225-298, 2003".

[10] L. Huiqi, O. Chutatape, "Automated feature extraction in color retinal images by a model based approach, IEEE Transactions on Biomedical Engineering, Vol.51, pp.246-254, 2004.

[11] C. Marin o, G. Penedo, M. Penas, J. Carreira, F. Gonzalez "Personal authentication using digital retinal images", Pattern Analysis and Applications, Vol.9, pp.21-33, 2006.

[12] C. Köse, C. Ikibas, "A personal identification system using retinal vasculature in retinal fundus images", Expert Systems with Applications, Vol.38, pp.13670-13681, 2011.

[13] B. Cassin, S.A.B. Solomon, Dictionary of Eye Terminology, 2nd ed., Triad Publishing Company, 1990.

[14] Foracchia M, Grisan E, Ruggeri A,"Luminosity and contrast normalization in retinal images", Med Imaging Anal, Vol.9, pp.179-90, 2005.

[15] Ehsan S, "Luminosity and contrast normalization in color retinal images based on the standard reference image", Proc. SPEI 9784, Medical Imaging, 2016.

[16] "GopalDutt Joshi, "Colour Retinal Image Enhancement based on Domain Knowledge", Proc. of the IEEE Sixth Indian Conference on Computer Vision, Graphics and Image Processing (ICVGIP 2008),pp. 591-598, 2008, Bhubaneswar, India".

[17] Shin DS, Javornik NB, Berger JW, "Computer-assisted, interactive fundus image processing for macular drusen quantitation", Ophthalmology, Vol.106, pp.1119-25, 1999.

[18] Leandro JJG, Soares JVB, Cesar Jr RM, Jelinek HF, "Blood vessels segmentation in non-mydriatic images using wavelets and statistical classifiers, In: XVI Brazilian symposium on computer graphics and image processing, p.262-9, 2003.

[19] Rapantzikos K, Zervakis M, Balas K, "Detection and segmentation of drusen deposits on human retina: potential in the diagnosis of age-related macular degeneration", Med Imaging Anal,Vol.7, pp.95-108, 2003.

[20] Thani, "Color retinal image enhancement by Rayleigh contrastlimited adaptive histogram equalization", 14th International Conference on Control, Automation and Systems (ICCAS), 2014.

[21] T.Jintasuttisak, S.Intajag, "Color Retinal Image Enhancement by Rayleigh Contrast-Limited Adaptive Histogram Equalization”, 
14th International Conference on Control, Automation and Systems, Korea, pp. 22-25, 2014

22] N.S.Datta, "A new contrast enhancement method of retinal images in Diabetic Screening System", IEEE 2nd International Conference on Recent Trends in Information Systems (ReTIS), 2015

[23] Saikat Kumar Shome, "Enhancement of Diabetic Retinopathy Imagery Using Contrast Limited Adaptive Histogram Equalization", International Journal of Computer Science and Information Technologies, Vol.2, pp.2694-2699, 2011.

[24] J. A Stark, "Adaptive Image Contrast Enhancement Using Generalizations of Histogram Equalization", IEEE Transactions on Image Processing, Vol.9, pp.889-894, 2000.

[25] Ni M Noor, N Abdul Khalid, Mohd Ali, Alice D Numpang, "Fish Bone Impaction Using Adaptive Histogram Equalization (AHE)", Second International Conference on Computer Research and Development, IEEE Computer Society, 2010

26] F. ter Haar, "Automatic localization of the optic disc in digita colour images of the human retina," Thesis, Utrecht University, Utrecht, the Netherlands, 2005.

[27] Sopharak, B. Uyyanonvara, S. Barman, and T. H. Williamson, "Automaticdetection of diabetic retinopathy exudates from nondilated retinal images using mathematical morphology methods," Comput. Med. Imag. Graph.,Vol. 32, pp. 720-727, 2008

28] T.Walter, J.-C. Klein, P.Massin, and A. Erginay, "A contribution of image processing to the diagnosis of diabetic retinopathydetection of exudates in color fundus images of the human retina," IEEE Transactions onMedical.Imaging, Vol. 21, pp.1236-1243, 2002

[29] S. Lu and J.-H.Lim, "Automatic optic disc detection from retinal images by a line operator," IEEE Trans. Biomed.Eng., vol. 58, no. 1, pp. 88-94, 2011

30] S. Lu, "Accurate and efficient optic disc detection and segmentation by a circular transformation," IEEE Trans. Med. Imag., vol. 30, no. 12, pp. 2126-2133, 2011.

[31] Youssif, A. Z. Ghalwash, and A. S. Ghoneim, "Optic disc detection from normalized digital fundus images by means of a vessels' direction matched filter," IEEE Trans. Med. Imag., vol. 27, no. 1, pp. 11-18, 2008

32] R. Rangayyan, X. Zhu, F. Ayres, and A. Ells, "Detection of the optic nerve head in fundus images of the retina with Gabor filter and Phase Portrait analysis.” J. Digital Imag., vol. 23, no. 4, pp 438-453, 2010.

[33] D. Welfer, J. Scharcanski, C. M. Kitamura, M. M. Dal Pizzol, L. W. B. Ludwig, and D. R. Marinho, "Segmentation of the optic disk in color eye fundus images using an adaptive morphological approach," Comput. Biol.Med., vol. 40, no. 2, pp. 124-137, 2010.

[34] M. Mendonca, A. Sousa, L. Mendonca, and A. Campilho, "Automatic localization of the optic disc by combining vascular and intensity information," Comput. Med. Imag. Graph., Officia J. Comput. Med. Imag. Soc., vol. 37, nos. 5/6, pp. 409-417, 2013.

[35] M. Goldbaum, S. Moezzi, A. Taylor, S. Chatterjee, J. Boyd, E. Hunter, and R. Jain, "Automated diagnosis and image understanding with object extraction, object classification, and inferencing in retinal images," in Proc. Int. Conf. Image Process. Sep 1996, vol. 3, pp. 695-698.

[36] M. Niemeijer, M. D. Abrmoff, and B. van Ginneken, "Fast detection of the optic disc and fovea in color fundus photographs," Med. Image Anal., vol. 13, no. 6, pp. 859-870, 2009.

[37] S. Morales, V. Naranjo, J. Angulo, and M. Alcaniz, "Automatic detection of optic disc based on PCA and mathematica morphology," IEEE Trans. Med. Imag., vol. 32, no. 4, pp. 786796, 2013.

[38] Giachetti, L. Ballerini, E. Trucco, and P. Wilson, "The use of radial symmetry to localize retinal landmarks",Comput.Med.Imag. Graph Vol.37, No.56, pp. 369-376, 2013.

[39] Giachetti, L. Ballerini, and E. Trucco, "Accurate and reliable segmentation of the optic disc in digital fundus images," J. Med. Imag., vol.1, no.2, pp. 024001-1-024001-11, 2014.

[40] D. Zhang andY. Zhao, "Novel accurate and fast optic disc detection in retinal images with vessel distribution and directional characteristics," IEEE J. Biomed. Health Informat., 2014.

41] D. Marin, M. E. Gegundez-Arias, A. Suero, and J. M. Bravo, "Obtaining optic disc center and pixel region by automatic thresholding methods on morphologically processed fundus images," Comput. Methods Prog.Biomed., vol. 118, no. 2, pp. $173-185,2015$

[42] Aquino, M. Gegundez-Arias, and D. Marin, "Detecting the optic disc boundary in digital fundus images using morphological, edge detection, and feature extraction techniques," IEEE Trans. Med. Imag., vol. 29, no. 11, pp. 1860-1869, 2010.
[43] R. J. Qureshi, L. Kovacs, B. Harangi, B. Nagy, T. Peto, and A Hajdu, "Combining algorithms for automatic detection of optic disc and macula in fundus images," Comput. Vis. Image Understanding, vol.116, no.1, pp.138-145, 2012.

[44] J. Cheng, J. Liu, Y. Xu, F. Yin, D.Wong, N.-M.Tan, D. Tao, C.-Y. Cheng, T. Aung, and T. Y. Wong, "Super pixel classification based optic disc and optic cup segmentation for glaucoma screening," IEEE Trans.Med. Imag., vol. 32, no. 6, pp. 10191032, Jun. 2013.

[45] [E. Trucco,H.Azegrouz, and B. Dhillon, "Modeling the tortuosity of retinal vessels: Does caliber play a role?" IEEE Trans. Biomed. Eng., vol. 57, no. 9, pp. 2239-2247, Sep. 2010.

[46] W. Lotmar, A. Freiburghaus, and D. Bracher, "Measurement of vessel tortuosity on fundus photographs," Albrecht von GraefesArchivF urKlinische and ExperimentelleOphthalmologie, vol. 211, no. 1, pp. 49-57, 1979.

[47] C. Heneghan, J. Flynn, M. OKeefe, and M. Cahill "Characterization of changes in blood vessel width and tortuosity in retinopathy of prematurity using image analysis," Med. Image Anal., vol. 6, no. 4, pp. 407-429, 2002.

[48] W. E. Hart, M. Goldbaum, B. C`ot'e, P. Kube, and M. R. Nelson, "Measurement and classification of retinal vascular tortuosity," Int. J. Med.Informatics, vol. 53, no. 2, pp. 239-252, 1999.

[49] M. Perez, A. Highes, A. Stanton, S. Thorn, N. Chapman, A Bharath, and K. Parker, "Retinal vascular tree morphology: A semi-automatic quantification," IEEE Trans. Biomed. Eng., vol. 49, no. 8, pp. 912-917, 2002

[50] "P. Benitez-Aguirre,M. E.Craig,M.B. Sasongko, A. J. Jenkins, T.Y.Wong, J. J. Wang, N. Cheung, and K. C. Donaghue", "Retina vascular geometry predicts incident retinopathy in young people with type 1 diabetes a prospective cohort study from adolescence," Diabetes Care, vol. 34, no. 7, pp. 1622-1627, 2011".

[51] "E. Bullitt, G. Gerig, S. Pizer, W. Lin, and S. Aylward", "Measuring tortuosity of the intracerebral vasculature from MRA images," IEEE Trans. Med. Imag., vol. 22, no. 9, pp. 1163-1171, 2003".

[52] "E. Grisan, M. Foracchia, and A. Ruggeri”, "A novel method for the automatic grading of retinal vessel tortuosity," IEEE Trans. Med. Imag., vol. 27, no. 3, pp. 310-319, 2008",

[53] "M. Patasius, V. Marozas, A. Lukosevicius, and D. Jegelevicius", "Evaluation of tortuosity of eye blood vessels using the integral of square of derivative of curvature," in Proc. EMBEC, 2005, vol. 5 , pp. 20-25"

[54] "R. Crosby-Nwaobi, L. Z. Heng, and S. Sivaprasad", "Retinal vascular calibre, geometry and progression of diabetic retinopathy in type 2 diabetes mellitus," Ophthalmologica, vol. 228 , no. 2, pp. 84-92, 2012',

[55] "M. B. Sasongko, T. Y. Wong, T. T. Nguyen, R. Kawasaki, A. J. Jenkins, J. Shaw, C. Robinson, and J. J. Wang", "Serum apolipoproteins are associated with systemic and retinal micro vascular function in people with diabetes," Diabetes, vol. 61, no. 7, pp. 1785-1792, 2012”.

[56] "K. Chandrinos, M. Pilu, R. Fisher, and P. Trahanias", "Image processing techniques for the quantification of atherosclerotic changes," UK: University of Edinburgh, DAI Res. Paper, 1998.

[57] “K. G. Goh, W. Hsu, M. Li Lee, and H. Wang”, "Adris: An automatic diabetic retinal image screening system," Stud. Fuzziness Soft Comput., vol. 60, pp. 181-210, 2001

[58] "A. Kaupp, H. Toonen, S. Wolf, K. Schulte, R. Effert, D. Meyerebrecht, and M. Reim", "Automatic evaluation of retinal vessel width and tortuosity in digital fluorescein angiograms," Investigative Ophthalmol. Visual Sci., vol. 32, no. 4, pp. 863-863, 1991

[59] "F. Ghadiri, H. Pourreza, T. Banaee, andM. Delgir", "Retinal vessel tortuosity evaluation via circular hough transform," in Proc. 18th Iranian Conf. Biomed. Eng., Dec. 2011, pp. 181-184

[60] "F. Ghadiri, S. Zabihi, H. Pourreza, and T. Banaee", "A novel method for vessel detection using contourlet transform," in Proc.Nat.Conf.Commun., Feb. 2012, pp. 1-5.

[61] "D. K. Wallace, S. F. Freedman, and Z. Zhao", "Evolution of plus disease in retinopathy of prematurity: Quantification by roptool," Trans. Amer. Ophthalmological Soc., vol. 107, pp. 47-52, 2009

[62] "S. Roychowdhury, D. D. Koozekanani , Keshab K and Parhi", "Blood Vessel Segmentation of Fundus Images by Major Vesse Extraction and Sub-Image Classification", in Biomedical and Health Informatics, IEEE Journal, pp. 2168-2194, (2014).

[63] "D. Marn, A. Aquino, M. Emilio Gegndez-Arias and J. Manuel Bravo", "A new supervised method for blood vessel segmentation in retinal images by using gray-level and moment invariants-based features", in Medical Imaging, IEEE Transactions,vol.30,no.1, pp 146-158, (2011) 
[64] "K. Sun, Z. Chen, S. Jiang, and Y. Wang", "Morphological multistage enhancement, fuzzy filter and watershed for vascular tree extraction in angiogram," Journal of medical systems," in Journal of medical systems,vol.35, pp. 811-824, 2011.

[65] "M.S. Miri and A. Mahloojifar", "An approach to localize the retinal blood vessels using bit planes and center- line detection", in Computer methods and programs in biomedicine,vol.108, pp. 600616, 2012.

[66] "M.M. Fraz et al.", "Retinal image analysis using curvelet transform and multi-structure elements morphology by reconstruction," in Biomedical Engineering, IEEE Transactions,vol.58, pp. 1183-1192, 2011.

[67] Y. Hou, "Automatic Segmentation of Retinal Blood Vessels Based on Improved Multiscale Line Detection," in Journal of Computing Science and Engineering, vol.8, pp. 119-128, 2014.

[68] "Demambita Kumar", "Retinal blood vessel segmentation using matched filter and Laplacian of Gaussian", International Conference on Signal Processing and Communications (SPCOM), 2016.

[69] "HooshiarZolfagharnasab and Ahmad Reza Naghsh-Nilchi", "Cauchy Based Matched Filter for Retinal Vessels Detection", Journal of Medical Signals and Sensors, Vol.4, no.1, pp.1-9, 2014.

[70] Xianjing Meng, "A framework for retinal vasculature segmentation based on matched filters", Biomedical Engineering, 2015.

[71] "S. Chaudhuri, S. Chatterjee, N. Katz, M. Nelson, M. Galbanum", "Detection of blood vessels in retinal images using twodimensional matched filters", IEEE Transactions on Medical Imaging, Vol.8,pp.263-269, 1989.

[72] "M. Al-Rawi, M. Qutaishat, M. Arrar", "An improved matched filter for blood vessel detection of digital retinal images", Computers in Biology and Medicine, Vol.37, pp.262-267, 2007.

[73] C. Yao, H.-j. Chen, "Automated retinal blood vessels segmentation based on simplified PCNN and fast 2D-Otsu algorithm", Journal of Central South University of Technology, Vol.16, pp. 640-646, 2009.

[74] Jingdan Zhang, "Blood Vessel Segmentation of Retinal Images Based on Neural Network", Springer International Publishing, pp.11-17, 2015.

[75] Rahebi J, "Retinal blood vessel segmentation with neural network by using gray-level co-occurrence matrix-based features", J Med Syst, Vol.38, no.8, 2014.

[76] Pawel, "Segmenting Retinal Blood Vessels With Deep Neural Networks, IEEE Transactions on Medical Imaging, Vol.35, no.11, Nov. 2016.

[77] R. Nekovei, "Back-propagation network and its configuration for blood vessel detection in angiograms", IEEE Transactions on Neural Networks, Vol.6 no.1, Page 64-72, January 1995.

[78] Lili Xu and Shuqian Luo, "A novel method for blood vessel detection from retinal images", BioMedical Engineering, 2010.

[79] Marlin M, Shan B, "Robust and Efficient Segmentation of Blood Vessel in Retinal Images using Gray level Texture features and Fuzzy SVM", Journal of Biomedical Pharmacol, Vol.8, no.1, 2015.

[80] L. Xu, S. Luo, "A novel method for blood vessel detection from retinal images”, Bio-Medical Engineering, Vol.14, 2010.

[81] X. You, Q. Peng, Y. Yuan, Y.-m.Cheung, J. Lei, "Segmentation of retinal blood vessels using the radial projection and semisupervised approach”, Pattern Recognition, Vol.44, pp.2314-2324, 2011.

[82] Songyuan Tang, "Retinal Vessel Segmentation Using Supervised Classification Based on Multi-Scale Vessel Filtering and Gabor Wavelet", Journal of medical imaging and health informatics, Vol.5, 2015.

[83] Qinmu Peng, "Retinal Blood Vessels Segmentation Using the Radial Projection and Supervised Classification", International Conference on Pattern Recognition, 2010.

[84] "Y.A. Tolias, S.M. Panas", "A fuzzy vessel tracking algorithm for retinal images based on fuzzy clustering", IEEE Transactions on Medical Imaging,Vol.17, pp.263-273, 1998.

[85] "J. Ng, S.T. Clay, S.A. Barman, A.R. Fielder, M.J. Moseley, K.H. Parker, C. Paterson", "Maximum likelihood estimation of vessel parameters from scale space analysis", Image and Vision Computing, Vol.28, pp.55-63, 2010.

[86] “A. Simó, E. de Ves", "Segmentation of macular fluorescein angiographies: A statistical approach", Pattern Recognition, Vol.34, pp.795-809, 2001.

[87] "G.B. Kande, P.V. Subbaiah, T.S. Savithri", "Unsupervised fuzzy based vessel segmentation in pathological digital fundus images", Journal of Medical Systems, Vol.34, pp.849-858, 2009.
[88] "S. Salem, N. Salem, A. Nandi", "Segmentation of retinal blood vessels using a novel clustering algorithm (RACAL) with a partial supervision strategy", Medical and Biological Engineering and Computing,Vol.45, pp.261-273, 2007.

[89] "Cree MJ, Olson JA, McHardy KC, Sharp PF, Forrester JV”, “A fully automated comparative microaneurysm digital detection system",Vol.11, pp.622-628, 1997.

[90] "Goatman KA, Cree MJ, Olson JA, Sharp PF, Forrester JV", "Automated measurement of microaneurysm turnover", Invest Ophthalmol Vis Sci, Vol.44, pp.5335-41, 2003.

[91] Dr. Seetaiah Kilaru, Hari Kishore K, Sravani T, Anvesh Chowdary L, Balaji T "Review and Analysis of Promising Technologies with Respect to fifth Generation Networks", 2014 First International Conference on Networks \& Soft Computing, ISSN:978-1-47993486-7/14,pp.270-273,August2014.

[92] Meka Bharadwaj, Hari Kishore "Enhanced Launch-Off-Capture Testing Using BIST Designs" Journal of Engineering and Applied Sciences, ISSN No: 1816-949X, Vol No.12, Issue No.3, page: 636-643, April 2017.

[93] N Bala Dastagiri, Kakarla Hari Kishore "Reduction of Kickback Noise in Latched Comparators for Cardiac IMDs" Indian Journal of Science and Technology, ISSN No: 0974-6846, Vol No.9, Issue No.43, Page: 1-6, November 2016.

[94] Murali, K Hari Kishore, D Venkat Reddy "Integrating FPGAs with Trigger Circuitry Core System Insertions for Observability in Debugging Process" Journal of Engineering and Applied Sciences, ISSN No: 1816-949X, Vol No.11, Issue No.12, page: 2643-2650, December 2016.

[95] Mahesh Mudavath, K Hari Kishore "Design of CMOS RF FrontEnd of Low Noise Amplifier for LTE System Applications Integrating FPGAs" Asian Journal of Information Technology, ISSN No: 1682-3915, Vol No.15, Issue No.20, page: 4040-4047, December 2016

[96] P Bala Gopal, K Hari Kishore, B.Praveen Kittu "An FPGA Implementation of On Chip UART Testing with BIST Techniques", International Journal of Applied Engineering Research, ISSN 0973-4562, Volume 10, Number 14 , pp. 34047 34051, August 2015

[97] S Nazeer Hussain, K Hari Kishore "Computational Optimization of Placement and Routing using Genetic Algorithm" Indian Journal of Science and Technology, ISSN No: 0974-6846, Vol No.9, Issue No.47, page: 1-4, December 2016.

[98] N Bala Gopal, K Hari Kishore "Analysis of Low Power Low Kickback Noise in Dynamic Comparators in Pacemakers" Indian Journal of Science and Technology, ISSN No: 0974-6846, Vol No.9, Issue No.44, page: 1-4, November 2016. 